\title{
\begin{tabular}{l|l|l|l|l}
\hline $\mathrm{M}$ & $\mathrm{R}$ & $\mathrm{S}$ & Internet Journal of & Nitride Semiconductor Research \\
\hline
\end{tabular}
}

Volume 2, Article 24

\section{Transient four wave mixing experiments on GaN}

\author{
R. Zimmermann, M. Hofmann, D. Weber, J. Möbius, A. Euteneuer, W. W. Rühle \\ Fachbereich Physik und Zentrum für Materialwissenschaften, Marburg, Germany \\ E. O. Göbel \\ Physikalisch-Technische Bundesanstalt, Braunschweig, Germany \\ B.K. Meyer \\ I. Physics Institute, Justus-Liebig-University Giessen \\ H. Amano, I. Akasaki \\ Department of Electrical and Electronic Engineering, Meijo University
}

This article was received on June 10, 1997 and accepted on September 8, 1997.

\begin{abstract}
We present four wave mixing experiments on GaN. We find an intrinsic homogeneous broadening of the A-exciton of $1.67 \mathrm{meV}$. A pronounced beating with a period of $0.52 \mathrm{ps}$ is observed at excitation energies between the $\mathrm{A}$ - and the B-exciton and corresponds to an energy splitting of $7.98 \mathrm{meV}$ of $\mathrm{A}$ and B-exciton.
\end{abstract}

\section{Introduction}

Group III - Nitrides based on GaN have recently been shown to have an enormous potential for optoelectronic applications in the visible up to ultraviolet spectral range. Light emitting diodes (LEDs) [1] and laser diodes (LDs) [2] [3] have been developed. Excitonic contributions have been shown to play an important role in the emission processes of these devices [4]. The study of excitonic resonances with linear optical experiments is often difficult. In particular, photoluminescence spectra are often dominated by bound excitons and free exciton resonances are difficult to determine. In contrast, transient degenerate four wave mixing (FWM) experiments are a well suited, nonlinear optical method to determine as well the excitonic resonance as its dephasing time and thereby the homogeneous broadening of the resonance [5]. Additionally, small energy splittings [6] can be determined with high precision even in the case of large inhomogeneous broadening. In this article, we present transient FWM studies on wurtzite GaN and determine the dephasing time of the A-exciton as well as the energy splitting of the A- and B-exciton.

\section{Experimental details}

We use a frequency doubled Kerr-lens modelocked Ti:sapphire laser with pulses of $\approx 180$ fs full width at half maximum. The laser beam is divided into two parts which are temporally delayed with respect to each other using a variable delay stage. The FWM experiment is performed in the two-pulse self diffraction configuration in reflection geometry [5] shown schematically in Figure 1. The two beams are focussed onto the sample with a lens. The coherent interaction of the two pulses with the wavevectors $\vec{k}_{1}$ and $\vec{k}_{2}$ generates there a third order nonlinear polarization giving rise to the FWM signal, which is coherently emitted into the phase - matched direction $2 \vec{k}_{2}-\vec{k}_{1}$. We detect the signal in reflection since the transmitted beam is scattered by the rough surface of the substrate. The FWM signal is measured spectrally and temporally integrated as a function of the time delay $\tau$ between the two exciting pulses using a photomultiplier. 
The sample is a MOCVD grown, about $3 \mu \mathrm{m}$ thick layer of GaN deposited on a (0001) sapphire substrate with the c-axis perpendicular to the surface plane [7]. It is kept in a Helium flow cryostat at a temperature of $\approx 10 \mathrm{~K}$.

\section{Homogeneous linewidth of the A-exciton}

First, we tune the energy of the exciting laser to the A-exciton resonance $(3.494 \mathrm{eV})$ and measure the FWM signal as a function of time delay in order to investigate the dephasing time of the A-exciton. A typical FWM transient is shown in Figure 2. The decay of the FWM signal towards positive time delay yields the dephasing time $T_{2}$ of the exciton. We obtain a $T_{2}$ of 0.58 ps according to: $T_{2}=2 \cdot \tau_{\text {decay }}$, where $\tau_{\text {decay }}$ is the single exponential decay time. The homogeneous broadening $\Gamma$ ) of the A-exciton for these excitation conditions is then $2.3 \mathrm{meV}$, according to $\Gamma=2 \mathrm{~T}_{\mathrm{T}} / \mathrm{T}_{2}$ (we assume here the limit of homogeneous broadening since the sample does not exhibit a Stokes shift and the linewidths obtained from linear optical measurements are comparable to the homogeneous broadening that we obtain).

We perform measurements of the homogeneous broadening as a function of both, temperature and exciton density, respectively, in order to separate the effects of exciton - phonon and exciton - exciton scattering. The homogeneous broadening $\Gamma_{\text {hom }}$ exhibits a linear dependence on temperature $T$ and on exciton density $n_{x}$ [5] [8]: $\Gamma_{\text {hom }}\left(T, n_{x}\right)=\Gamma_{00}+\gamma_{p h}{ }^{T}+\gamma_{x x} a_{b}{ }^{3} E_{b} n_{x}$, at low temperatures when acoustic phonon scattering is the dominant scattering mechanism varying with temperature and at low densities when exciton-exciton scattering varies with density. The intrinsic homogeneous broadening $\Gamma_{00}$ accounts for scattering of excitons with crystal defects, $\gamma_{\mathrm{ph}}$ and $\gamma_{\mathrm{xx}}$ are a measure for scattering of excitons with acoustic phonons and scattering with other excitons, respectively. Finally, $a_{B}=3.6 \mathrm{~nm}$ is the Bohr radius of the exciton and $E_{B}=20 \mathrm{meV}[9]$ is its binding energy. The temperature dependence ( $\mathrm{a}$, for two different excitation densities) and the density dependence (b), for two different temperatures) of $\Gamma_{\text {hom }}$ are shown in Figure 3a. From a linear fit to all our data, we find $\Gamma_{00}=$ $1.73 \mathrm{meV}, \gamma_{\mathrm{ph}}=8.2 \mu \mathrm{eV} / \mathrm{K}$, and $\gamma_{x x}=2$.2. Stanley Pau and coworkers found on a different sample a value for $\Gamma_{00}$ which is about 4 times smaller than our value. [9] They also find an about three times stronger density dependence and no temperature dependence of the homogeneous broadening. However, it should be noted that propagation effects (exciton-polaritons) might play an important role for the measured decay times of the FWM signal [10]. We expect, however, that for our experiments which are performed in reflection geometry these effects are less important than in transmission [11]. The influence of propagation effects will be subject of future studies.

Second, we study the influence of the photon energy of the exciting laser on the FWM transients. A set of FWM transients for different excitation energies between $3.491 \mathrm{eV}$ and $3.515 \mathrm{eV}$ is shown in Figure 4. For excitation energies between the A- and B-exciton resonance, the transients exhibit strong modulations with a period of $0.52 \mathrm{ps}$. These modulations originate from the coherent excitation of both the A-and B-excitons with the spectrally broad laser pulse. The modulations are caused either by polarization interference or by real quantum beats between the A-exciton and the B-exciton. In both cases, the beat period $\tau_{A B}$ of the modulations directly provides the energy splitting $\Delta_{A B}$ of A-exciton and B-exciton: $\Delta E_{A B}=h / \tau_{A B}$, and we obtain accordingly a value of $\Delta \mathrm{E}_{\mathrm{AB}}=7.98 \mathrm{meV}$ in our sample. This value is in the same range as well as the energy splitting of A- and B- excitons obtained on similar [7] and also on different samples [12].

\section{Conclusions}

In conclusion, we present first transient four wave mixing studies on GaN with wurtzite structure. We find an intrinsic homogeneous broadening of $1.67 \mathrm{meV}$ for the A-exciton. A pronounced beating is observed in the four wave mixing transients when the energy of the exciting laser is tuned between the A-exciton and the B-exciton. The beat period yields an energy splitting of $7.98 \mathrm{meV}$ of the A- and the B-exciton.

\section{References}

[1] Shuji Nakamura, Masayuki Senoh, Naruhito Iwasa, Shin-ichi Nagahama, Appl. Phys. Lett. 67, 1868-1870 (1995).

[2] S. Nakamura, M. Senoh, S. Nagahama, N. Iwasa, T. Yamada, T. Matsushita, Y. Sugimoto, H. Kiyoku, Appl. Phys. Lett. 69, 1477-1479 (1996). 
[3] I. Akasaki, S. Sota, H. Sakai, T. Tanaka, M. Koike, H. Amano, Electron. Lett. 32, 1105-1106 (1996).

[4] Y. Narukawa, Y. Kawakami, M. Funato, S. Fujita, S. Fujita, S. Nakamura, Appl. Phys. Lett. 70, 981-983 (1997).

[5] R. Hellmann, M. Koch, J. Feldmann, S. T. Cundiff, E. O. Göbel, D. R. Yakovlev, A. Waag, G. Landwehr, Phys. Rev. B 48, 2847 (1993).

[6] M. Koch, G. von Plessen, J. Feldmann, E. O. Göbel, Chem. Phys. 120, 367 (1996).

[7] D Volm, K Oettinger , T Streibl, D Kovalev, M Ben-Chorin, J Diener, BK Meyer, J Majewski, L Eckey , A Hoffman, H Amano, I Akasaki, K Hiramatsu, T Detchprohm, Phys. Rev. B 53, 16543-16550 (1996).

[8] E. J. Mayer, N. T. Pelekanos, J. Kuhl, N. Magnea, H. Mariette, Phys. Rev. B 51, 17263 (1995).

[9] S. Pau, J. Kuhl, F. Scholz, V. Haerle, unpublished (1997).

[10] A. Schulze, A. Knorr, S. W. Koch, Phys. Rev. B 51, 10601 (1995).

[11] V. Kutzer, B. Lummer, R. Heitz, A. Hoffmann, I. Broser, E. Kurtz, D. Hommel, J. Cryst. Growth 159, 776 (1996).

[12] W. Shan, B. D. Little, A. J. Fischer, J. J. Song, B. Goldenberg, W. G. Perry, M. D. Bremser, R. F. Davis, Phys. Rev. B 54, 16369 (1996).

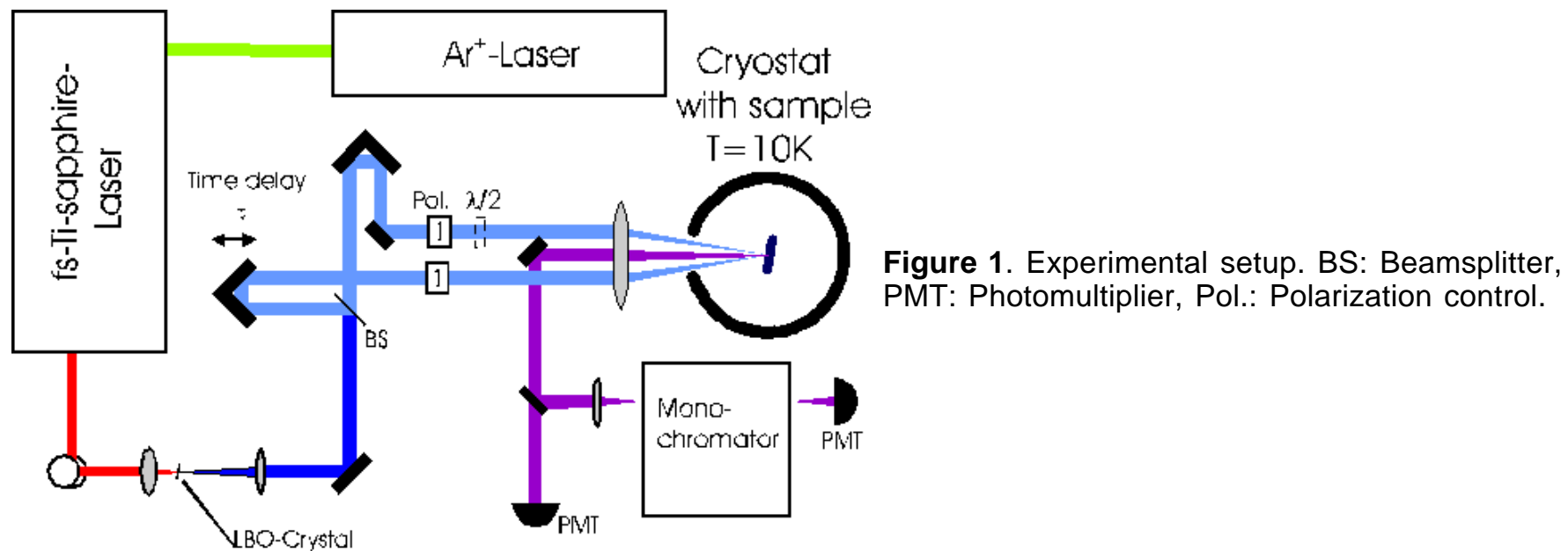




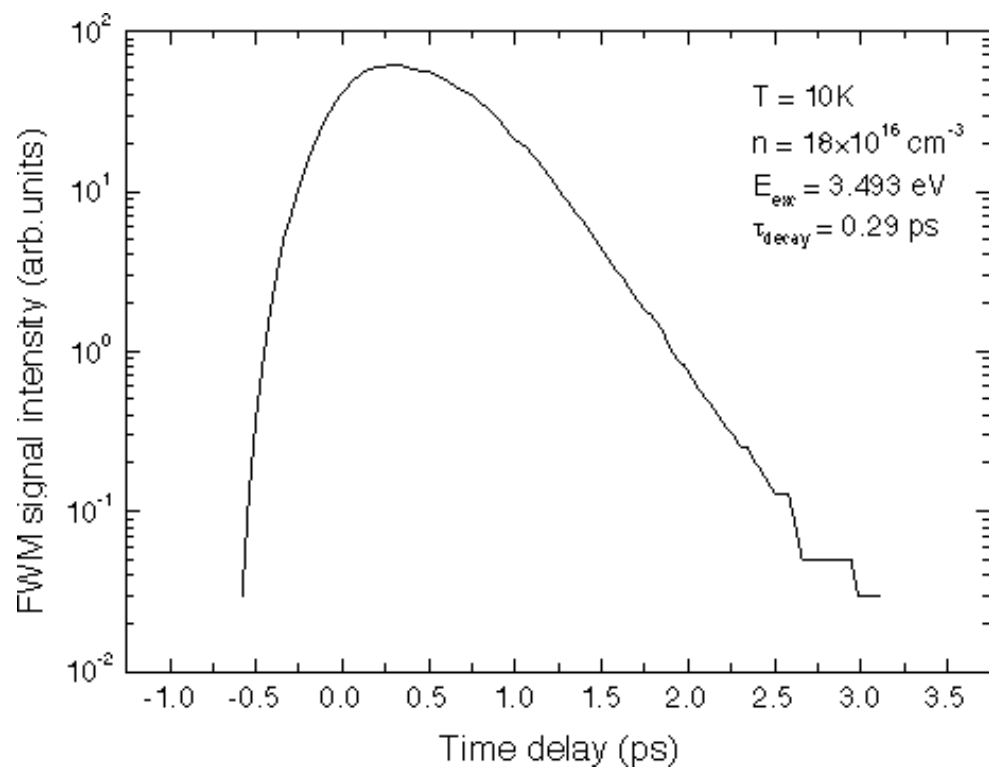

Figure 2. Time-integrated FWM transient for an excitation energy of $3.493 \mathrm{eV}$.

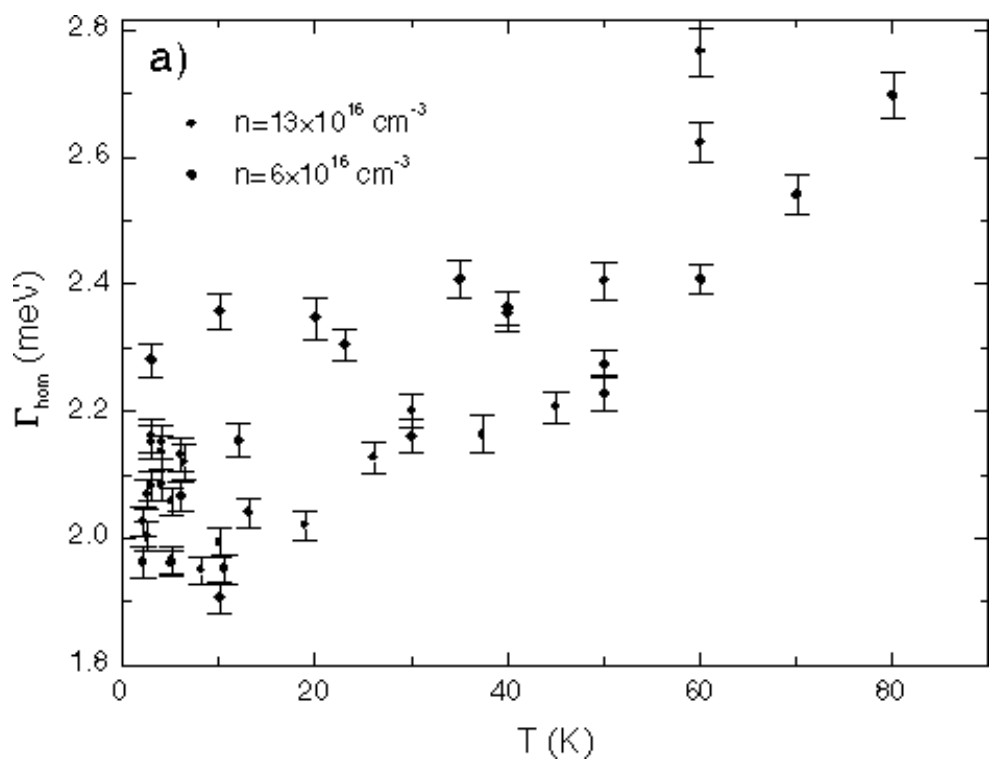

Figure 3a. Homogeneous broadening of the A-exciton as a function of temperature for two different excitation densities.

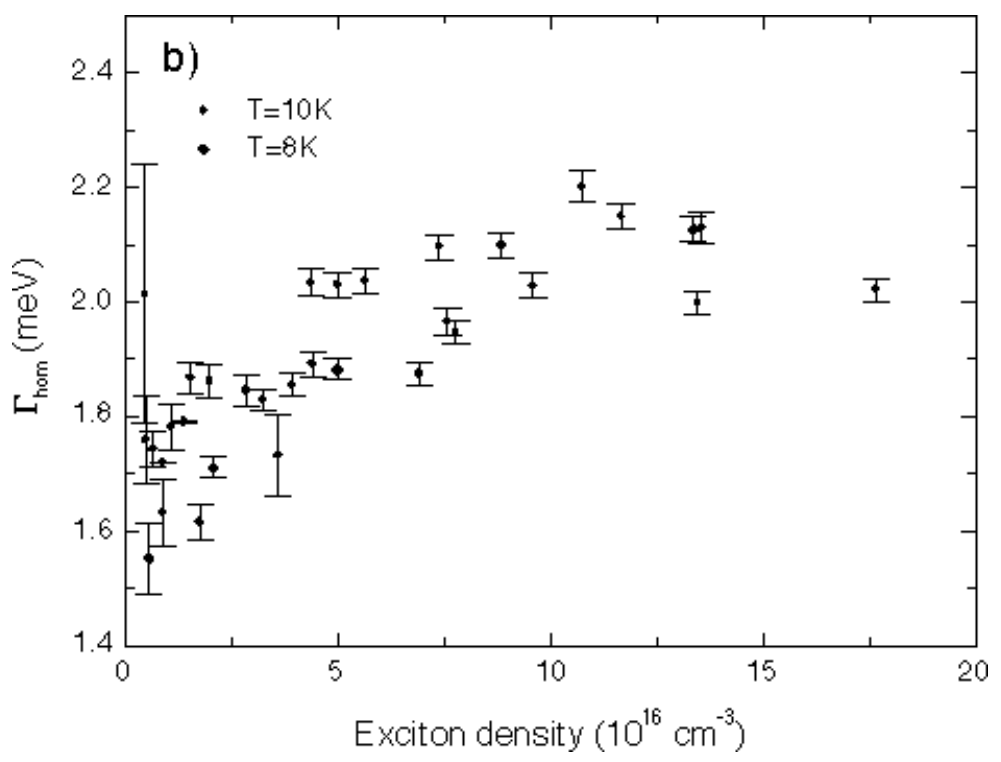

Figure $3 \mathbf{b}$. Homogeneous broadening of the A-exciton as a function of excitation density for two different temperatures. 


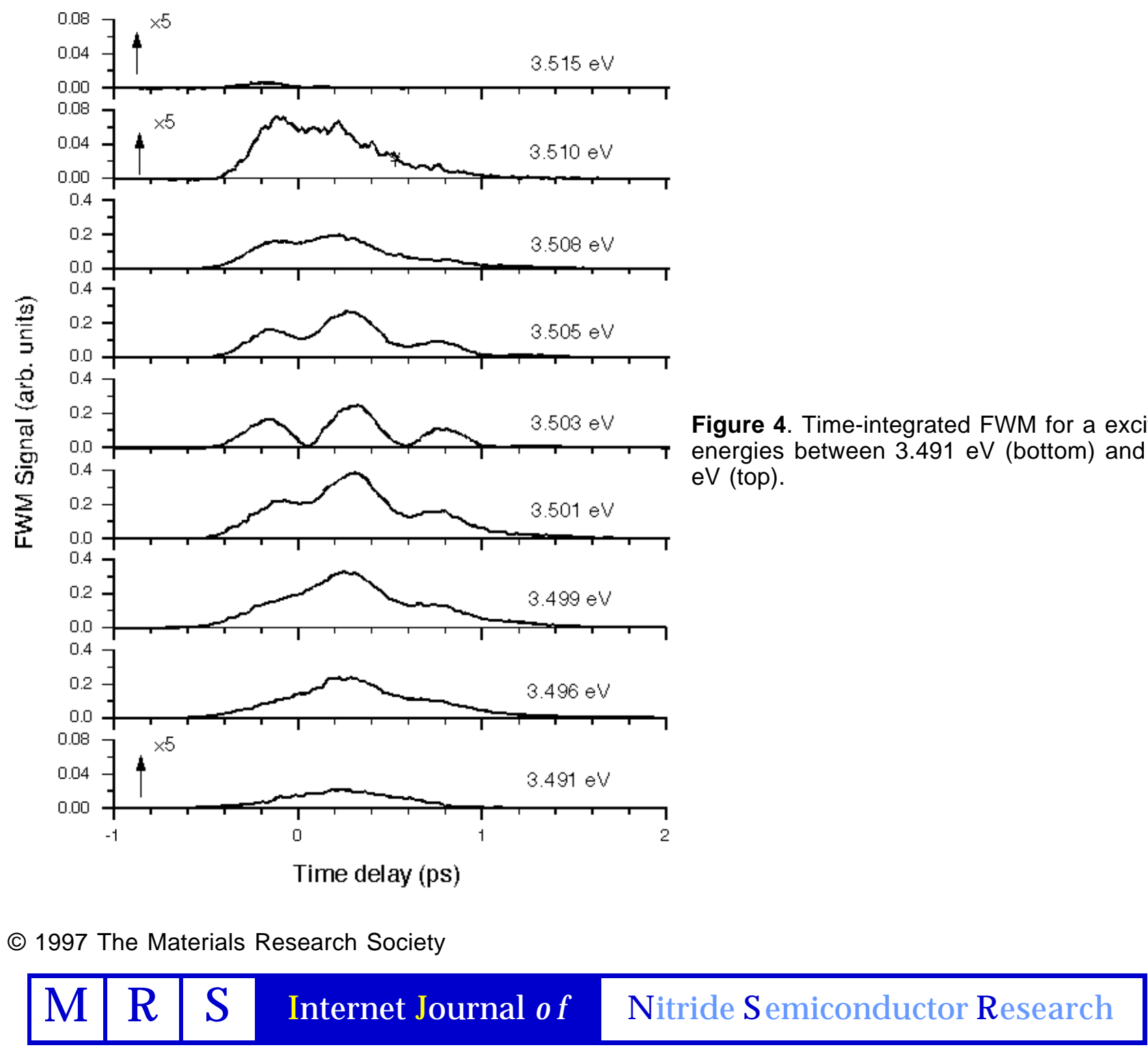

